\title{
MICROHANDLING AND ASSEMBLY: THE PROJECT ASSEMIC
}

\author{
Ana Almansa ${ }^{1}$, Silvia Bou ${ }^{2}$, Domnita Fratila ${ }^{3}$ \\ l,2,3 Austrian Research Centres Seibersdorfs GmbH, 2444 Seibersdorf, Austria
}

\begin{abstract}
Mechatronic competences represent a strong component in Microsystems Technologies, but very especially in Microhandling and -assembly, a field with challenging requirements. The Research and Training Network "Advanced Methods and Tools for Handling and Assembly in Microtechnology" (ASSEMIC) addresses this research field at a European scale. This paper presents aspects of the ASSEMIC project and some results achieved in its frame.
\end{abstract}

Key words: Training, Microassembly, Microtechnology, Microhandling

\section{INTRODUCTION}

The project "Advanced Methods and Tools for Handling and Assembly in Microtechnology" (ASSEMIC) was launched in January 2004, with the aim of addressing the broad and challenging research area of microhandling and assembly in the frame of a Marie Curie - Research and Training Network (RTN) ${ }^{1}$.

With a consortium constituted by 14 participants from 10 different countries and a training and research schedule corresponding to more than 500 person months, the project ASSEMIC is among the largest Research and Training Networks funded by the European Commission. More than 30 foreign researchers, specifically appointed for the project, will be trained during its four years duration ${ }^{2}$. The members of ASSEMIC consortium are:

1. ISAS - TU Wien (Austria)

2. FSRM (Switzerland)

3. ARC Seibersdorf research GmbH (Austria)

4. IMT (Romania)

5. Politechnika Warszawska-WUT (Poland) 
6. Uninova (Portugal)

7. University of Oldenburg (Germany)

8. Fundacion Robotiker (Spain)

9. FORTH (Greece)

10. Progenika Biopharma S.A. (Spain)

11. CCLRC - RAL (Great Britain)

12. Fraunhofer Gesellschaft - ILT (Germany)

13. Scuola Superiore Sant'Anna (Italy)

14. Nascatec (Germany).

The project is structured in several workpackages. A brief description of them content is given below ${ }^{1}$ :

WP 1. Micropositioning: Positioning stages and elements with integrated sensors and feedback control, autonomous and mobile systems, microrobotics.

WP 2. Microhandling: Tools and methods for handling in different environments (normal room conditions, clean room, vacuum, fluids) and applications.

WP 3. Microassembly: Innovative tools, strategies and alternative approaches for efficient micro-assembly.

WP 4. Automation for industrial production: Including production chains, quality assurance, test and characterization issues, etc.

WP 5. Know-how management: Technology transfer and dissemination.

\section{FIRST RESEARCH RESULTS}

\subsection{Micropositioning}

The first workpackage is dedicated to technology for high accuracy positioning in microhandling applications. Different applications and potential uses have been considered in this workpackage such as: RAL's functionalized cantilever technology, which is applicable for novel Atomic Force Microscopy (AFM) probes, and optical position sensors developed by Uninova's. 


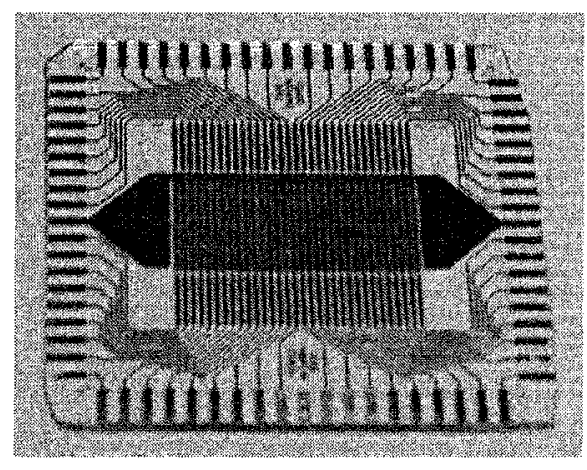

Figure 1. Uninova's position sensor

\subsection{Microhandling}

The second workpackage deals with the development of technology for microhandling, which involves also a number of other further micromanipulation operations not directly related to assembly of MEMS, among others concerning biological and medical applications.

Examples of this are microsurgery, cell manipulation and detection and handling of biochemical macromolecules. Within the task devoted to the development of Advanced Microhandling tools, research has been done on different types of microgrippers, as well as special fabrication methods for such micro-grippers. Nascatec has reported an electrostatic microgripper and performed additional mechanical simulations by means of Finite Element Analysis.

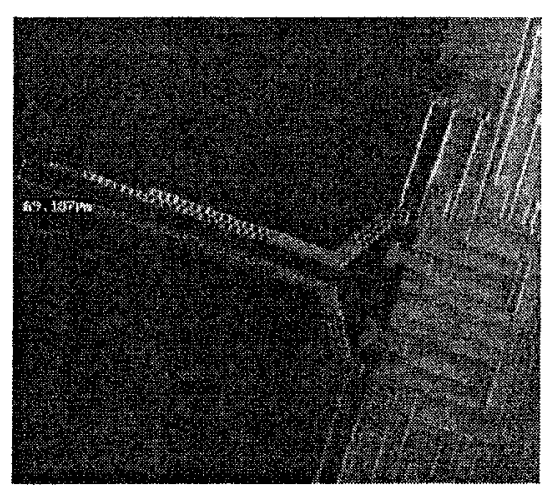

Figure 2. Electrostatic gripper (Nascatec) 
While Scuola Superiore Sant'Anna has developed microgrippers by using a technology a technology for PST-actuated tools by using Shape Deposition Manufacturing (SDM), provided by previous collaboration with University of Uppsala in Sweden, Seibersdorf research has explored the combination of LIGA (Lithographie Galvanik Abformung) and PIM (Powder Injection Moulding) for the production of microgrippers.
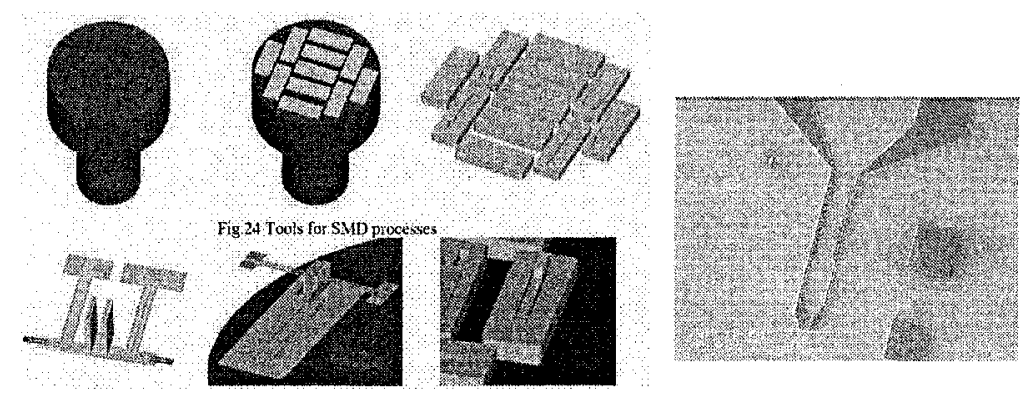

Figure 3. Left: Tools for SDM processes (SSSA); Right: gripper photoresist structure on wafer (Seibersdorf)

Beyond the work on development of gripping tools, an extensive study has been performed by ARC Seibersdorf research, with contribution of Politechnika Warszawska -WUT, concerning strategies for handling of microcomponents. Concerning adhesion and material issues related to microhandling processes, Politechnika Warszawska-WUT has proposed two solutions for special intelligent coating with controllable adhesion. Furthermore, Scuola Superiore Sant'Anna performed some experiments to compare theoretical and real adhesion forces between sample and needle fingertip under different environmental conditions (normal and dry environment).

As regards microhandling applications, several application possibilities have been proposed and analyzed by the ASSEMIC participants, in order to test the tools and methods developed in the project. One of them, as reported by the University of Oldenburg, is the manipulation of TEM-lamella in the semiconductor industry.

The University of Oldenburg has also adapted and tested additional tools for manipulation of nanowires. They have done several experiments for gripping nanowires and for bonding them with the help of Electron Beam Deposition (EBD), with satisfactory results.

Another field of interest explored in Workpackage 2 comprises biological and medical applications for micromanipulation, with contributions from partners as Progenika and CCLRC (functionalization of biomedical cantilever based sensors), IMT-Bucharest (Surface Acoustic Wave micro- 
agitation device for DNA hybridization) and SSSA (mechanical and chemical measurement from vessels).

\subsection{Microassembly}

In a first approach to the development of Innovative Tools for assembly of microparts, results available at Fraunhofer Gesellschaft / Institute für Lasertechnik on integrated tools for gripper and joining of components, will serve as a basis for further work in the ASSEMIC project.

Another technology of interest for assembly of MEMS is micro-gluing. A study was done concerning adhesive used in Micro- opto- electromechanical systems (MOEMS) and microtechniques, as well as the different techniques of gluing and dispensing, including automated dispensing, UV-curing adhesives and those used for encapsulation ${ }^{4}$.

Advanced processes for efficient microassembly, such as design for assembly, parallel assembly or self-assembly processes have been analysed and reported in a state of the art performed by Politechnika Warszawska WUT and Seibersdorf.

Further, work related to the assembly, testing and improvement of the $4 \times 4$ and $8 \times 8$ cross connector switches for optical fibres was done in cooperation between FSRM and the University of Neuchatel. After the conception of an adequate assembly strategy, among other tasks, a driver PCB, a Visual Basic program for switching the 64 mirrors, and a new high voltage source were developed.

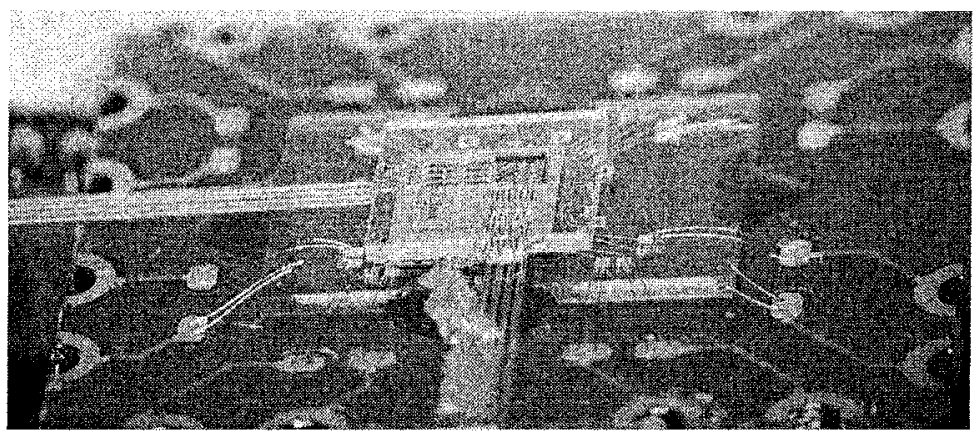

Figure 4. Side view of $4 \times 4$ packaged cross connection switch for optical fiber (FSRM/University of Neuchatel) 


\section{FURTHER WORK}

This paper has presented main research achievements and activities performed in the ASSEMIC Network during the one and a half year. During the next years, further joint research is planned in the fields of microrobotics (miniaturized autonomous mobile platforms) and advanced control systems for intelligent manipulation, as well as human haptic interfaces. Research on biological and medical applications of micromanipulation will be extended with experiments for operation in special environments and conditions, such as vacuum, normal room conditions and fluids. Furthermore, work done in tools and processes for micro-assembly will be complemented with the analysis of alternative approaches to hybrid assembly, in comparison with monolythical integration. Finally, a new workpackage will deal with automation of micro-assembly for industrial production of MEMS and covering automated handling, test and characterization of assembled Microsystems.

\section{ACKNOWLEDGMENT}

This paper and the described work have been made possible thanks to the financial support provided by the Marie Curie Programme under the 6th Framework Programme of the European Commission through the Research and Training Network "Advanced Methods and Tools for Handling and Assembly in Microtechnology" (ASSEMIC). The work described in this paper has been performed by the 14 institutions participating in the ASSEMIC Network. The authors would like to thank permanent staff and appointed fellows working in this project at these institutions, for their excellent work and co-operation.

\section{REFERENCES}

1. European Commission, "Marie Curie Research and Training Networks (RTN) Handbook" 2nd Edition, December 2003.

2. A. Almansa, S. Bou, W. Brenner, A. Locher: "A European Research and Training Network for Advanced Post-graduate Education", Proceedings of the IEEE International Conference on Industrial Technology; Hammamet; Tunesia, 2004. ISBN:0-7803-8663-9.

3. M. Fernandes, M. Vieira, I. Rodrigues, R. Martins. "Large area image sensing structures based on a-SiC: a dynamic characterization", Sensors and Actuators A, 113, (3), 2004, pp. 360-364.

4. S. Bou, A. Almansa, N. Balabanava, "Handling Processes in Microsystems Technology", submitted for publication at AIM 2005. 